\section{Aborto provocado: redução da frequência e gravidade das complicações. Consequência do uso de misoprostol?}

Daniela Fornel de Oliveira Silva 1

Aloísio José Bedone 2

Aníbal Faúndes 3

Arlete Maria dos Santos Fernandes 4

Verônica Gomes Alencar de Lima e Moura 5

\section{Artificial abortion: is reduction in the frequency and severity of complications a result of the use of misoprostol?}

1,2,4 Departamento de Ginecologia e Obstetrícia. Faculdade de Ciências Médicas. Universidade Estadual de Campinas. Campinas, SP, Brasil.

3 Centro de Pesquisas em Saúde Reprodutiva de Campinas (CEMICAMP). Caixa Postal 6181. CEP: 13.083-970. Campinas, SP, Brasil. E-mail: afaundes@unicamp.br

5 Secretaria Municipal de Saúde de Campinas. Campinas, SP, Brasil.

\section{Resumo}

Objetivos: verificar a frequência e a gravidade das complicações por abortos provocados e suas possíveis associações com o uso de misoprostol.

Métodos: estudo de corte transversal. Durante dez meses aplicou-se uma lista de verificação (critérios da World Health Organization) a todas as 543 mulheres internadas por aborto em dois hospitais na cidade de Campinas, São Paulo. Àquelas classificadas como aborto possível, provável ou certamente provocado foi aplicado também um questionário.

Resultados: dentre todas as mulheres internadas, 259 (48\%) foram classificadas como aborto possivel, provável ou certamente induzido e responderam ao questionário; 25 mulheres declararam a indução do aborto e, destas, nove referiram uso de misoprostol. Complicações infecciosas e hemorrágicas ocorreram respectivamente em $10 \%$ e $13 \%$ das 259 mulheres. As que usaram misoprostol se complicaram menos que as que usaram outros métodos, porém essa diferença não foi estatisticamente significativa, talvez pela baixa freqüência de complicações.

Conclusões: os dados mostram redução da freqüencia e da gravidade das complicações do aborto, mas não permitem avaliar o papel do misoprostol.

Palavras-chave Aborto induzido, Misoprostol 


\section{Introdução}

A World Health Organization (WHO) define aborto inseguro como um procedimento para interromper uma gestação não planejada, realizado por indivíduos que não possuem as habilidades necessárias ou num ambiente sem o mínimo dos padrões médicos preconizados, ou ambos, o que representa risco significativo para a saúde e para a vida das mulheres. ${ }^{1,2}$ De aproximadamente 50 milhões de abortos provocados que ocorrem anualmente no mundo, em torno de 19 milhões correspondem a abortos inseguros. ${ }^{2}$

O aborto inseguro é causa importante de mortalidade materna $a^{2,3}$ e coloca em risco principalmente as mulheres dos países em desenvolvimento, onde o aborto é restrito pela lei, e nos países onde, apesar de legalmente permitido, o aborto seguro não é de fácil acesso.4-7 Estima-se que 13\% de todas as mortes maternas no mundo sejam decorrentes do aborto inseguro, e os maiores percentuais são encontrados na América Latina (17\%) e Sudeste da Ásia $(19 \%) .1,2$

A mortalidade e a morbidade maternas relacionadas ao aborto provocado e inseguro dependem do acesso a métodos de menor risco para a interrupção da gestação. 8,9 As taxas de morbidade e hospitalização por aborto provocado têm diminuído desde os anos de 1990 em resposta ao uso de formas mais seguras de se realizar o aborto.1,10 $\mathrm{O}$ aumento do uso do misoprostol, substituindo métodos mais invasivos, provavelmente tem contribuído para a redução das complicações. $8,9,11,12$

No Brasil, o número de mulheres tratadas em hospitais públicos por complicações de abortos, caiu aproximadamente $28 \%$ em 13 anos. Entretanto, esse declínio foi maior entre os anos de 1992 e 1995.1 Desde então o número tem variado pouco. Estudos realizados em hospitais públicos do Rio de Janeiro, Fortaleza, Goiânia e Recife no início dos anos de 1990 demonstraram que a maioria das pacientes que relataram a indução do aborto usou misoprostol. Estas mulheres apresentaram complicações menos graves do que aquelas que utilizaram métodos invasivos e a frequência de complicações graves agudas foi menor entre elas. $8,11,13,14$

$\mathrm{Na}$ cidade de Campinas, a mortalidade materna por aborto, que era uma das principais causas de morte na década passada, ${ }^{15}$ parece ter-se reduzido consideravelmente, visto que foram registradas apenas três mortes no município no período compreendido entre 2000 e 2008.16

O uso do misoprostol, apesar de clandestino e ilegal no Brasil, pode ter tido um efeito de redução das complicações de aborto em ambientes de restrição legal ao procedimento. 8,9,11 Ressalta-se, entretanto, que a utilização descontrolada de um medicamento que provoca fortes contrações uterinas, podendo provocar até mesmo ruptura uterina, pode ser perigoso, ${ }^{17,18}$ principalmente quando se utilizam doses elevadas, como tem sido descrito em estudos brasileiros. ${ }^{19,20}$

Com o fim de avaliar a taxa de complicações graves como consequência de aborto provocado e sua relação com uso de misoprostol, foram entrevistadas mulheres internadas em duas maternidades que atendem clientela do Sistema Único de Saúde (SUS) da cidade de Campinas, Brasil.

\section{Métodos}

Durante dez meses, entre 2008 e 2009, foram convidadas a participar deste estudo de corte transversal todas as $\mathbf{5 4 3}$ mulheres internadas com diagnóstico de aborto em dois hospitais da cidade de Campinas, Brasil.

Estas pacientes foram submetidas a uma lista de verificação que continha os critérios da World Health Organization ${ }^{21}$ (três perguntas para a mulher sobre a gestação e quatro anotações obtidas do prontuário em relação à presença de sinais de infecção ou traumas durante a internação), a partir da qual as mulheres foram classificadas em abortos espontâneos ou em abortos possível, provável ou certamente induzidos.

Foram classificadas como "abortos possivelmente induzidos" as participantes que afirmaram não desejar a gravidez ou que disseram estar em uso de métodos contraceptivos (MAC) quando engravidaram ou apresentaram um ou mais dos seguintes sinais de infecção durante a internação: secreção com odor fétido ao exame ginecológico, febre (temperatura $\geq 38^{\circ} \mathrm{C}$ ) ou leucograma com número de bastões acima de 500. As mulheres que referiram que não desejavam a atual gravidez, que estavam em uso de MAC quando engravidaram e apresentaram pelo menos um dos sinais de infecção descritos acima foram classificadas como "abortos provavelmente induzidos". Foram classificadas como "abortos certamente induzidos", independentemente de ter apresentando qualquer um dos itens acima descritos, as mulheres que assumiram ter usado chá e/ou remédio e/ou outra coisa para descer a menstruação e/ou apresentaram sinais de manobras abortivas ao exame ginecológico.

Todas as mulheres classificadas como abortos induzidos ou suspeitos de induzidos responderam a um questionário estruturado pré-testado. Este 
continha perguntas objetivas e perguntas abertas cujas respostas foram codificadas, a respeito de características sociodemográficas, pessoais, antecedentes obstétricos, dados da atual gestação, da indução do aborto e de possíveis complicações. Requeria também anotações do prontuário em relação às possíveis complicações, as quais foram assim consideradas, "infecções leves": quando esteve presente algum dos sinais de infecção descritos anteriormente e/ou quando houve o diagnóstico de infecção limitada à cavidade uterina e que não precisou de outra intervenção além da evacuação de restos ovulares; "infecção grave": quando fo além da cavidade uterina e/ou necessitou de outra intervenção como por exemplo condutas cirúrgicas. As complicações hemorrágicas foram classificadas como "sangramento abundante": em grande quantidade ou com coágulos (este não havia como ser quantificado uma vez que os profissionais anotavam no prontuário apenas "pequena" ou "grande" quantidade, sem correlacionar com algo que o quantificasse); "sangramento abundante que requer transfusão": em grande quantidade, quando a mulher precisou ser transfundida; "sangramento muito abundante que necessitou de histerectomia": na presença de sangramento muito intenso que não pode ser controlado, exigindo a realização da histerectomia. As complicações traumáticas podiam ser: "lesões de vulva ou vagina", "desgarro de colo uterino", "perfuração do útero" e "lesão de órgãos vizinhos".

Os dados foram coletados após a realização da curetagem, próximo ao momento da alta hospitalar por enfermeiras treinadas para a pesquisa. As entrevistas foram realizadas no próprio hospital, na presença apenas da paciente e da entrevistadora. Nenhuma das mulheres convidadas se recusou a participar do estudo

Foi obtido o consentimento informado verbal das mulheres uma vez que o Comitê de Ética dispensou o uso do consentimento informado escrito e assinado, dada a ilegalidade do aborto induzido.

Para a análise dos dados, aplicou-se o quiquadrado de Pearson, qui-quadrado com correção de Yates ou o teste exato de Fisher. Foram considerados estatisticamente significativos os valores de $p<0,05$. O programa utilizado para as análises foi o SPSS for Windows v.17.0.

\section{Resultados}

Das 543 mulheres internadas com diagnóstico de aborto, cinco não tiveram suas entrevistas concluídas por diferentes motivos (quadros psiquiátricos ou interrupção da entrevista seguida de alta hospitalar da paciente antes do término da coleta de dados). Dentre as 538 mulheres restantes, 259 foram identificadas como mulheres que possivelmente, provavelmente ou certamente provocaram o aborto: a maior parte $(85,7 \%)$ correspondeu ao primeiro grupo, 11 $(4,3 \%)$ a provavelmente induzido e $26(10,0 \%)$ a aborto certamente provocado. Destas, 25 declararam ter usado algum método para a indução do aborto e uma apesar de não ter declarado, apresentou sinais de manobras abortivas ao exame ginecológico (lesões de vulva ou vagina)

Pouco mais da metade das 259 mulheres tinha idade entre 20 e 29 anos (54,83\%), escolaridade até o segundo grau $(57,2 \%)$, companheiro fixo $(61 \%)$ e dois terços referiram gestações anteriores $(67,2 \%)$.

Sinais de infecção foram notados em 35 mulheres $(13,5 \%)$ e 26 tiveram complicações hemorrágicas (10\%); ambas as complicações ocorreram em maior proporção entre as mulheres que assumiram a indução do aborto (Tabela 1). Nenhuma das participantes do estudo apresentou infecção grave ou sangramento abundante que terminasse em histerectomia.

Tabela 1

Percentual de complicações infecciosas e hemorrágicas em pacientes que declararam ou não o uso de algum método para induzir o aborto. Campinas, Brasil. 2008-2009.

\begin{tabular}{lcccc}
\hline Complicações detectadas & \multicolumn{2}{c}{$\begin{array}{c}\text { Declarou ter induzido } \\
(\mathbf{n = 2 5})\end{array}$} & $\begin{array}{c}\text { Negou ter induzido } \\
(\mathbf{n}=\mathbf{2 3 4 )}\end{array}$ \\
\cline { 2 - 5 } & $\mathrm{n}$ & $\%$ & $\mathrm{n}$ & $\%$ \\
\hline Complicação infecciosa & 8 & 32,0 & 27 & 11,5 \\
Complicação hemorrágica & 6 & 24,0 & 20 & 0,010 \\
& & & & 0,027 \\
\hline
\end{tabular}

* Teste exato de Fisher. 
Os métodos que as 25 mulheres declararam ter utilizado para interrupção da gravidez foram o misoprostol em nove casos (36\%) e outros métodos em 16 casos (64\%). Em apenas um caso foi declarado o uso de sonda intra-uterina e manobras de evacuação. Nos demais casos, as mulheres relataram uso de chás ou medicamentos (chá de arruda, chá de buxinha, chá de folha de laranja, chá de pimenta-do-reino, chá de maconha, chá de boldo, chá de arruda e canela, inalação com buxinha, ergotrate, anticoncepcional de emergência). Aquelas que referiram o uso de misoprostol apresentaram um menor percentual de complicações (tanto infecciosas quanto hemorrágicas), entretanto esta diferença não teve significância estatística (Tabela 2).

A maior parte das mulheres usou a dose de misoprostol recomendada de 800 microgramas $(\mathrm{mcg})$. Apenas uma mulher usou ( $600 \mathrm{mcg})$ e três usaram $1200 \mathrm{mcg}$, porém apenas uma delas usou mais de 800 mcg como dose unitária.

Em relação à forma de obtenção do misoprostol, duas mulheres conseguiram com uma amiga, outras duas conseguiram na farmácia e as outras cinco conseguiram com o próprio parceiro, com uma prima, via internet, por telefone e sozinha (numa casa de ervas).

O preço pago por três a seis comprimidos de misoprostol variou de $\mathrm{R} \$ 240,00$ a $\mathrm{R} \$ 400,00$ (aproximadamente 120 a 200 dólares), com o preço de cada comprimido variando entre $\mathrm{R} \$ 40,00$ a $\mathrm{R} \$$
133,00 (20 a 66 dólares).

Oitenta por cento das mulheres que referiram não desejar a gravidez estavam em uso de métodos contraceptivos quando engravidaram.

Não houve diferenças significativas quanto à idade, escolaridade, número de gestações, filhos nascidos vivos e filhos atualmente vivos entre as mulheres com aborto possivelmente induzido e o grupo de mulheres com aborto provável e certamente induzido. $\mathrm{O}$ percentual de mulheres com aborto provável ou certamente induzido foi maior entre as mulheres com história de aborto e entre as mulheres com três ou mais nascidos vivos ou vivos atualmente, mas essas diferenças não foram estatisticamente significativas.

A porcentagem de aborto provável e certamente induzido foi mais de duas vezes e significativamente maior entre as mulheres sem união estável que entre aquelas com parceiro fixo $(p=0,010)$ (Tabela 3$)$. Da mesma forma, a porcentagem de mulheres que apresentaram complicações hemorrágicas foi duas vezes e meia superior entre as mulheres sem união estável $(15,8 \%)$ que entre as mulheres com parceiro fixo $(6,3 \%)$. Essa diferença foi estatisticamente significativa $(p=0,023)$ (Tabela 4$)$. Não se observou nenhuma outra diferença significativa quando se analisaram as características sociodemográficas e os antecedentes obstétricos com relação à incidência ou não de complicações infecciosas ou hemorrágicas.

Tabela 2

Percentual de complicações infecciosas e hemorrágicas entre pacientes que usaram misoprostol e entre pacientes que usaram outros métodos. Campinas, Brasil. 2008-2009.

\begin{tabular}{|c|c|c|c|c|c|}
\hline \multirow[t]{2}{*}{ Complicações } & \multicolumn{2}{|c|}{$\begin{array}{l}\text { Referiu uso de misoprostol } \\
\qquad(n=9)\end{array}$} & \multicolumn{2}{|c|}{$\begin{array}{l}\text { Referiu uso de outros métodos } \\
\qquad(n=16)\end{array}$} & \multirow[t]{2}{*}{$p^{*}$} \\
\hline & $\mathrm{n}$ & $\%$ & $\mathrm{n}$ & $\%$ & \\
\hline Infecciosa & $2 *$ & 22,0 & 6 & 38,0 & ns \\
\hline Hemorrágica & $1 *$ & 11,0 & 5 & 31,0 & ns \\
\hline
\end{tabular}

* Teste exato de Fisher; ns = não significativo. 
Percentual de abortos possivelmente induzidos e de abortos provável e certamente induzidos entre mulheres com relação estável e mulheres sozinhas. Campinas, Brasil. 2008-2009.

\begin{tabular}{|c|c|c|c|c|c|c|}
\hline \multirow{2}{*}{$\begin{array}{l}\text { Classificação do } \\
\text { abortamento }\end{array}$} & \multicolumn{2}{|c|}{ Casada/vive junto } & \multicolumn{2}{|c|}{ Solteira/separada/viúva } & \multirow[t]{2}{*}{ Total } & \multirow[t]{2}{*}{$p^{*}$} \\
\hline & $\mathrm{n}$ & $\%$ & $\mathrm{n}$ & $\%$ & & \\
\hline Possivelmente induzido & 143 & 90,5 & 79 & 78,2 & 222 & \\
\hline Provavelmente/certamente induzido & 15 & 9,5 & 22 & 21,8 & 37 & 0,010 \\
\hline Total & 158 & 100,0 & 101 & 100,0 & 259 & \\
\hline
\end{tabular}

*teste qui-quadrado com correção Yates.

\section{Tabela 4}

Percentual de complicações hemorrágicas entre mulheres com relação estável e entre mulheres sozinhas. Campinas, Brasil. 2008-2009.

\begin{tabular}{lccccc}
\hline \multirow{2}{*}{$\begin{array}{l}\text { Complicações } \\
\text { hemorrágicas }\end{array}$} & \multicolumn{2}{c}{ Casada/vive junto } & Solteira/separada/viúva & \multirow{2}{*}{ Total } \\
\cline { 2 - 4 } & $\mathrm{n}$ & $\%$ & $\mathrm{n}$ & $\%$ & \\
\hline Sim & 10 & 6,3 & 16 & 15,8 & 26 \\
Não & 148 & 93,7 & 85 & 84,2 & 233 \\
Total & 158 & 100,0 & 101 & 100,0 & 259
\end{tabular}

*teste qui-quadrado com correção Yates.

\section{Discussão}

Nossos resultados mostram que a proporção de abortos classificados como possível, provável ou certamente induzidos entre as mulheres internadas foi similar à descrita na literatura brasileira na década passada $(48 \%), 8,13$ mas a taxa de complicações foi inferior $(13,5 \%$ das mulheres com sinais de infecção e $10 \%$ com complicações hemorrágicas comparadas, por exemplo, às $19,1 \%$ e $39,4 \%$ de estudo anterior, respectivamente). 8

Estes resultados estariam de acordo com os dados do Sistema de Informação de Mortalidade (SIM) da Secretaria Municipal de Saúde de Campinas que encontrou 80 casos de mortes maternas na cidade no período de 2000 a 2008 , sendo que apenas três foram comprovadamente consequência de aborto (4\%). 16

Chama a atenção que neste estudo apenas 25 mulheres declararam ter provocado a interrupção da gravidez $(9,65 \%$ das mulheres classificadas como abortos induzidos ou suspeitos). Há duas décadas, no início dos anos de 1990, vários estudos brasileiros encontraram porcentagens muito mais altas de mulheres que assumiram ter induzido seus abortos: em pesquisas realizadas no Rio de Janeiro, em Goiânia, em Fortaleza e no Recife no início dos anos de 1990 , essa porcentagem foi de $50 \%, 57 \%, 31 \%$ e $23 \%$, respectivamente. $8,11,13,19$ A baixa porcentagem observada em nossa pesquisa talvez pudesse ser explicada pela ampliação do planejamento familiar com consequente redução do número de gestações não planejadas e dos abortos induzidos. Entretanto a porcentagem de abortos classificados como suspeitos de induzidos, não se apresentou reduzida frente aos mesmos estudos, o que nos faz considerar a possibilidade de simples negativa das mulheres a dar essa informação. Sabemos que as mulheres tendem a omitir a informação sobre prática de aborto quando perguntadas diretamente sobre o assunto. $22-25$

Ao usar o misoprostol as mulheres apresentam menos complicações e procuram o hospital apenas para complementar o esvaziamento uterino, sem evidenciar sinais que permitam suspeitar que o aborto foi provocado 8 e portanto não vêem necessidade de assumir a indução. Assim, há provável subnotificação em nosso estudo: apenas nove das 
234 mulheres com suspeita de aborto induzido e das 25 que relataram ter provocado o aborto referiram o uso de misoprostol (3,5\%). Em outros três estudos, os percentuais de mulheres que usaram misoprostol foram $\quad 39 \% \quad$ (Goiânia), ${ }^{8} \quad 62 \% \quad$ (Recife) $^{11} \quad$ e $66 \%$ (Fortaleza). ${ }^{19}$

As mulheres parecem estar mais bem informadas sobre a dose apropriada de misoprostol que na década passada, visto que apenas uma delas tomou uma dose unitária um pouco acima da recomendada de $800 \mathrm{mcg}$. Em estudos anteriores, já citados acima, as doses muitas vezes eram bastante elevadas, entre 4000 e 16.800 mcg. ${ }^{19,20}$ Doses tão altas possuem riscos de efeitos secundários graves, de ruptura uterina na gestação do segundo trimestre e até do primeiro trimestre. 18

Por outro lado, o conceito de dividir a dose, entre a via oral e a via vaginal, parece estar profundamente arraigado, e as evidências da maior efetividade da via vaginal parecem não ter chegado ao conhecimento popular. 26

Nos estudos brasileiros citados, as complicações foram menos severas após o uso do misoprostol do que após o uso de outros métodos. $8,11,13$ Contudo, em nosso estudo, a comparação entre o grupo de mulheres que usou misoprostol com o grupo que utilizou outros métodos para indução do aborto não mostrou diferenças significativas na incidência de complicações infecciosas ou hemorrágicas, talvez devido à provável subnotificação das mulheres que usaram (mas não declararam) o misoprostol e não tiveram complicações.

Mulheres solteiras, separadas e divorciadas apresentaram uma maior frequência de abortos provável e certamente induzidos quando comparadas ao grupo de mulheres casadas e que vivem com os parceiros. Resultados semelhantes foram observados em estudo realizado em Fortaleza, em 1991, no qual se constatou que aproximadamente $70 \%$ das mulheres que induziram aborto eram solteiras. 14

Entre as demais variáveis analisadas, apesar das diferenças encontradas não terem atingido significação estatística, não surpreende que a porcentagem de abortos provável ou certamente induzidos tenha sido maior entre mulheres que já tiveram abortos. ${ }^{27}$ Da mesma forma, a proporção duas vezes maior de abortos provável e certamente induzidos entre as mulheres com mais filhos seguramente são uma expressão da decisão das mulheres que já têm o número desejado de filhos e que estão dispostas a qualquer medida, por difícil que ela seja, para limitar a família.

Não encontramos associação das variáveis sociodemográficas ou antecedentes obstétricos com a taxa de complicações infecciosas ou hemorrágicas (talvez pelos baixos percentuais das pacientes que apresentaram estas complicações), a não ser o estado marital: mulheres solteiras, separadas e divorciadas se correlacionaram a um maior índice de complicações hemorrágicas.

Não cabe contestar a redução das complicações graves e, consequentemente, da mortalidade relacionada ao aborto no município de Campinas. A baixa frequência de complicações em circunstâncias nas quais o número de abortos não vem diminuindo pode ser compatível com um maior uso de misoprostol como método de provocar os abortos, visto que diversos estudos têm mostrado que seu uso se acompanha de significativa diminuição das infecções pós-aborto. $8,9,11$ A diminuição das complicações de aborto e da gravidade das mesmas é comentário comum dos colegas de diversas cidades do Brasil e de outros países, onde o uso de misoprostol como medicamento abortivo está difundido. Ao considerar esta como a explicação principal da redução das complicações, significa que não se conseguiu que as voluntárias dessem informação correta sobre o uso deste medicamento, relatado por apenas nove delas. Estima-se, também, que o uso do misoprostol vem sendo feito em doses inferiores às efetivas para o completo esvaziamento uterino $28 \mathrm{ou}$ que as mulheres não esperem a eliminação completa dos restos ovulares uma vez que, como citado acima, não se observa diminuição do número de internações por aborto, mas sim o de complicações. 29

Pensamos que a conclusão mais importante deste estudo é justamente que se confirma o conceito generalizado de ter havido uma mudança na frequência e gravidade das complicações associadas à prática do aborto. Entretanto, não ter obtido informação verossímil das mulheres que participaram do estudo, prejudica verificar até que ponto o uso de misoprostol é responsável por essa redução, como observado em outros estudos.

Serão necessárias novas pesquisas, após reavaliar a forma de abordar estas pacientes, para se tentar que todas aquelas que induziram o aborto tenham coragem de declará-lo e de relatar o método utilizado. Além disso, se faz necessário repetir estudos mais acurados sobre mortalidade materna que permitam verificar se efetivamente a mortalidade materna associada ao aborto é tão mínima como aparece nas estimativas oficiais.

\section{Agradecimentos}

Agradecemos ao Centro de Pesquisas em Saúde Reprodutiva de Campinas (Cemicamp) todo apoio 
estrutural e análise estatística dos dados. Agradecemos também a Bem-Estar Familiar no Brasil (BEMFAM) e a Fundação de Amparo à Pesquisa do Estado de São Paulo (FAPESP), que

\section{Referências}

1. Grimes DA, Benson J, Singh S, Romero M, Ganatra B, Friday E. Unsafe abortion: the preventable pandemic. Lancet. 2006; 368 : 1908-19.

2. WHO (World Health Organization). Global and regional estimates of the incidence of unsafe abortion and associated mortality in 2003. 5 ed. Geneva; 2007.

3. Majlessi F, Forooshani AR, Shariat M. Prevalence of induced abortion and associated complications in women attending hospitals in Isfahan. East Mediterr Health J. 2008; 14: 103-9.

4. Kwast BE, Rochat RW, Kidane-Marian W. Maternal mortality in Addis Ababa, Ethiopia. Stud Fam Plann. 1986; 17: 288-301.

5. Castle MA, Likwa R, Whittaker M. Observations on abortion in Zambia. Stud Fam Plann. 1990; 21: 231-5.

6. Camp S. The impact of Mexico City policy on women and health care in developing countries. J Int Law Pol. 1987 20: 35-52.

7. Parpinelli MA, Faúndes A, Cecatti JG, Surita FGC, Pereira BG, Passini Jr R, Amaral E. Subnotificação da mortalidade materna em Campinas: 1992-1994. RBGO. 2000; 22: 2732 .

8. Viggiano MGC, Faúndes A, Borges AL, Viggiano ABF, Souza GR, Rebello I. Disponibilidade de misoprostol e complicações de aborto provocado em Goiânia. J Bras Ginecol. 1996; 106: 55-61.

9. Miller S, Lehman T, Campbell $\mathrm{M}$, Hemmerling A Anderson SB, Rodriguez H, Gonzalez WV, Cordero M Calderon V. Misoprostol and declining abortion-related morbidity in Santo Domingo, Dominican Republic: a temporal association. BJOG. 2005; 112: 1291-6.

10. Juarez F, Cabigon J, Singh S, Hussain R. The incidence of induced abortion in the Philippines: current level and recent trends. Int Fam Plan Perspect. 2005; 31: 140-9.

11. Faúndes A, Santos LC, Carvalho M, Gras C. Post-abortion complications after interruption of pregnancy with misoprostol. Adv Contracept. 1996; 12: 1-9.

12. Lafaurie MM, Grossman D, Troncoso E, Billings DL, Chavez S. Women's perspectives on medical abortion in Mexico, Colombia, Ecuador and Peru: a qualitative study. Reprod Health Matters. 2005; 13: 75-83.

13. Costa SH, Vessey MP. Misoprostol and illegal abortion in Rio de Janeiro, Brazil. Lancet. 1993; 341: 1258-61.

14. Coelho HLL, Teixeira AC, Santos AP, Forte EB, Morais SM, La Vecchia C, Tognoni G, Herxheimer FRCP. Misoprostol and illegal abortion in Fortaleza, Brazil. Lancet. 1993; 341: 1261-3. Erratum in: Lancet. 1993; 341: 1486

Recebido em 31 de julho de 2009

Versão final apresentada em 12 de julho de 2010

Aprovado em 28 de agosto de 2010 financiaram esta pesquisa. Somos gratos a todas as mulheres voluntárias entrevistadas que aceitaram nos relatar suas vivências.
15. Parpinelli MA, Faúndes A, Surita FGC, Pereira BG, Cecatti JG. Mortalidade materna na cidade de Campinas, no período de 1992 a 1994. RBGO. 1999; 21: 227-32.

16. SIM (Sistema de Informação de Mortalidade). Secretaria Municipal de Saúde de Campinas. [acesso 14 maio 2009]. Disponível em: http://tabnet.saude.campinas.sp.gov.br/ cgi/deftohtm.exe?sim/sim.def

17. Tang OS, Gemzell-Danielsson K, Ho PC. Misoprostol: pharmacokinetic profiles, effects on the uterus and sideeffects. Int J Gynaecol Obstet. 2007; 99 (Suppl 2): S160-7.

18. Kim JO, Han JY, Choi JS, Ahn HK, Yang JH, Kang IS, Song MJ, Nava-Ocampo AA. Oral misoprostol and uterine rupture in the first trimester of pregnancy: a case report. Reprod Toxicol. 2005;20 : 575-7.

19. Fonseca W, Misago C, Correa LL, Parente JAM, Oliveira FC. Determinantes do aborto provocado entre mulheres admitidas em hospitais em localidade da região Nordeste do Brasil. Rev Saúde Pública. 1996; 30: 13-8.

20. Costa SH. Commercial availability of misoprostol and induced abortion in Brazil. Int J Gynecol Obstet. 1998; 63 (Suppl. 1): S131-9.

21. World Health Organization. Task force on safety and efficacy of fertility regulating methods. Protocol for hospital based descriptive studies of mortality, morbidity related to induced abortion. Geneva; 1987.

22. Figa-Talamanca I. Illegal abortion: an attempt to assess its cost to the health services and its incidence in the community. Int J H Serv. 1986; 16: 375-89.

23. Bleek W. Lying informants: a fieldwork experience from Ghana. Pop Dev Rev. 1987; 13: 314-22.

24. Hardy E, Rebello I, Rodrigues T, Moares T.M. Aborto provocado: diferenças entre pensamento e ação. Rev Ginecol Obstet .1991; 2: 111-6.

25. Osis MJD, Hardy E, Faúndes A, Rodrigues T. Dificuldades para obter informações da população de mulheres sobre aborto ilegal. Rev Saúde Pública. 1996; 30: 444-51.

26. Faúndes A, Fiala C, Tang OS, Velazco A. Misoprostol for the termination of pregnancy up to 12 completed weeks of pregnancy. Int J Gynecol Obstet. 2007; 99: S172-7.

27. Heikinheimo O, Gissles M, Suhonen S. Age, parity, history of abortion and contraceptive choice affect the risk of repeat abortion. Contraception. 2008; 78: 149-54.

28. Weeks A, Faundes A. Misoprostol in obstetrics and gynecology. Int J Gynaecol Obstet. 2007; 99 (Suppl. 2): S156-9.

29. Bugalho A, Faúndes A, Jamisse L, Usfá M, Maria E, Bique C. Evaluation of the effectiveness of vaginal misoprostol to induce first trimester abortion. Contraception. 1996; 53: 244-6. 